\title{
GROUP THEORETIC CATEGORIZATION OF BIFURCATION MODES OF TRUSS DOME STRUCTURES
}

\author{
By Kiyohiro IKEDA*, Kunio TORIF* and Shogo MATSUSHITA***
}

\begin{abstract}
A study of potential bifurcation modes of dome structures is performed based on a group theoretic method for predicting the bifurcation modes. Potential bifurcation modes of a series of polygonal-shaped truss domes were advanced by investigating the subgroups of dihedral groups. These modes were classified into seven general categories and several rules regarding the presence of the modes were drawn. In addition, hierarchal structures of bifurcation modes were investigated. The information regarding bifurcation modes obtained in this manner was used to trace bifurcation buckling behavior of an eight-gonal truss dome. While an initial imperfection technique was used for tracing bifurcation buckling behavior, the information was employed to determine the initial imperfection modes which can initiate the bifurcation behavior.
\end{abstract}

Keywords: bifurcation buckling, symmetry, group theory, polygonal domes.

\section{INTRODUCTION}

Symmetry plays an important role in the field of structural engineering. Numerous structures have been constructed to hold a number of point and line symmetries. Such examples can be found literally everywhere, including a series of historic structures : the Colosseum in Rome, the Pyramids in Egypt, and the Blue Mosque in Istanbul. Dome structures can be set forth as a representative of modern structures with highly symmetric geometries. Dome structures, however, often exhibit bifurcation buckling behavior at the onset of a sharp reduction of load carrying capacities and drastic loss of symmetry ${ }^{1)}$. Naturally, it is of great engineering interest to evaluate bifurcation modes of dome structures and trace their bifurcation behavior.

Several analytical methods ${ }^{2)}$ 7) have been developed by a number of engineers for the purpose of tracing the behavior. These include initial imperfection ${ }^{21.3)}$, perturbation ${ }^{7)}$, and eigenvalue analysis ${ }^{6)}$ techniques. However, these techniques focused on the analytical tracing aspects of bifurcation behavior, while somewhat ignoring symmetric nature of bifurcation modes that should have a great influence on the behavior. Because of this, there exists only limited information regarding bifurcation modes, which has been obtained through case studies performed in a heuristic fashion ${ }^{1) \sim 7)}$.

By contrast, extensive mathematical studies on bifurcation behavior have revealed that such behavior is a process of losing symmetry. Dihedral groups, which can measure the level of symmetry of figures, are used to describe the symmetric nature of bifurcation modes. The bifurcation behavior has been found to be covariant with (analogous to) the groups.

\footnotetext{
* Member of JSCE Ph. D, Research Fellow, Technological University of Nagaoka, (Nagaoka, Niigata, Japan 940-21).

** Member of JSCE D Eng., Professor, Technological University of Nagaoka.

*** B Eng., Master Student, Technological University of Nagaoka.
} 
The previous papers ${ }^{8.9)}$ applied such a mathematical approach to the description of bifurcation modes of dome structures. Bifurcation buckling behavior of polygonal-shaped, reticulated, truss domes was proved to be covariant with (analogous to) a dihedral group, which is uniquely determined from the geometry of the dome and its loading conditions ${ }^{10) .11}$. Bifurcation modes of a hexagonal-shaped dome was advanced through an investigation of dihedral groups.

The objective of this paper is to develop a systematic and practical method for predicting bifurcation modes of dome structures with the use of dihedral groups. Potential bifurcation modes of a series of polygonal-shaped truss domes are expressed by means of the groups. A categorization of the modes is performed and general rules regarding the existence of modes are drawn. In addition, the information regarding bifurcation modes is utilized to select appropriate initial imperfection modes which can induce bifurcation buckling phenomena.

\section{GROUP THEORETIC METHOD FOR DESCRIBING SYMMETRY}

This section briefly reviews a group theoretic method for describing bifurcation behavior of dome structures that was advanced in the previous papers ${ }^{8.9)}$ and is directly employed for the purpose of discussion in this paper.

Isometries of a plane can be defined as one-to-one mappings on the Euclidian plane that preserve the distance between any two points in the plane ${ }^{12}$. Isometries are the products of reflections, translations and rotations. A figure in the Euclidian plane is stated more symmetric if the geometry of the figure can be preserved by more isometries.

Symmetry groups, made up of a set of isometries, have been extensively employed for representing symmetry of figures in mathematics ${ }^{12)}$. These group are utilized here to express the symmetry of bifurcation modes. The order of a group, equal to the number of the elements of the group, serves as an appropriate parameter to represent the level of symmetry.

The symmetry group of a regular $n$-gon $(n=3,4,5, \cdots)$ is called the dihedral group of a degree $n, D_{n}$. This group, whose order is equal to $2 n$, consists of the following $2 n$ isometric transformations in the $x^{-} y$ plane :

$\sigma_{j}$ and $\tau \sigma_{j} \quad j=1,2, \cdots, n$

where $\sigma_{j}$ is the $360 \cdot(j-1) / n$ degree clockwise rotation about the origin $\mathrm{O}$ and $\tau$ is the reflection in the $y$-axis ; the multiple of transformations denotes that the transformations are achieved in sequence from the right one to the left. The level of symmetry of a deformed configuration of the $n$-gon can be expressed by the number of elements preserving its geometry, or, in other words, by the order of its symmetry group.

Fujii ${ }^{10)}$.11) found that bifurcation behavior of a certain system is covariant with a symmetry group $G$ (G-covariant). Such behavior is a process of losing symmetry and analogous to the subgroup structure of the symmetry group. His findings with respect to a G-covariant system are summarized as : (1) The paths of a G-covariant system are characterized by their symmetry groups, which are subgroups of the group $\mathrm{G} ;(2)$ the path having the group $\mathrm{G}$ as its symmetry group is called a fundamental path ; (3) a path will preserve its symmetry group until a bifurcation point is reached; (4) when bifurcation paths branch from an equilibrium path at a bifurcation point with a single root, symmetry groups of the bifurcation paths are subgroups of the equilibrium path's symmetry group; (5) all the single critical points on the paths with the trivial symmetry group $\mathrm{E}$, in general, are stationary points of the loading parameter $f$, where $E$ is the group made up of the identity element $\sigma_{1}$.

Ikeda et al. ${ }^{9)}$ applied these findings to the description of bifurcation behavior of an $n$-gonal, reticulated, elastic, truss-dome (see Fig. 1) subjected to symmetric loadings applied at the nodes 1 through $n$. Its bifurcation behavior was proved to be covariant with the dihedral group $D_{n}$ and described by Fujii's findings. We concluded that potential bifurcation modes of a dome structure should be determined through the observation of subgroups of a dihedral group covariant with the behavior. 


\section{BIFURCATION MODES OF POLYGONAL-SHAPED TRUSS DOMES}

In order to assist the study of bifurcation behavior of dome structures, potential bifurcation modes of a series of polygonal-shaped truss domes are investigated here (see Fig. 1 for their typical configuration). As reported in Reference 9, deformational properties of these domes can be monitored by observing the subgroups of the group $D_{n}$, which is a symmetry group of a regular $n$-gon.

At first, a four-gonal dome shown in Fig. 2 is studied as a simple example. Potential bifurcation modes of this dome are characterized by the subgroups of the dihedral group $D_{4}$ :

$$
\begin{aligned}
& E=\left\langle\sigma_{1}\right\rangle \cdots \cdots C_{2}=\left\langle\sigma_{1}, \sigma_{3}\right\rangle \cdots \cdots C_{4}=\left\langle\sigma_{1}, \sigma_{2}, \sigma_{3}, \sigma_{4}\right\rangle \\
& D_{1}^{2 j-1}=\left\langle\sigma_{1}, \tau \sigma_{2 j-1}\right\rangle \cdots \cdots D_{1}^{2 j}=\left\langle\sigma_{1}, \tau \sigma_{2 j}\right\rangle \quad j=1 \text { or } 2 \\
& D_{2}^{1}=\left\langle\sigma_{1}, \sigma_{3}, \tau \sigma_{1}, \tau \sigma_{3}\right\rangle \cdots \cdots D_{4 / 2}=\left\langle\sigma_{1}, \sigma_{3}, \tau \sigma_{2}, \tau \sigma_{4}\right\rangle \\
& D_{4}=\left\langle\sigma_{1}, \sigma_{2}, \sigma_{3}, \sigma_{4}, \tau \sigma_{1}, \tau \sigma_{2}, \tau \sigma_{3}, \tau \sigma_{4}\right\rangle
\end{aligned}
$$

These subgroups were obtained with the aid of the following two steps : (1) obtain the subsets of the group; (2) check if each subset can become a group ${ }^{12}$. These steps should enable one to obtain all the subgroups of a group without fail.

The bifurcation modes related to these groups are schematically illustrated in Fig. 3 in terms of the typical deformed states of the nodes 1 through 4 of the dome. Multiple subgroups represented the same bifurcation mode; such cases can be interpreted that multiple bifurcation modes are degenerated into a single mode (mode degeneration) ${ }^{9}$. The highest-ordered subgroup among the subgroups, which can most accurately represent the symmetry of the mode, should be selected as its symmetry group. Consequently, bifurcation modes of the dome can be represented by the following five subgroups $: E, D_{1}^{2 j-1}, D_{1}^{2 j}, D_{4 / 2}$, and $D_{4}$.

The group $\mathrm{E}$ denotes a completely asymmetric bifurcation mode, which cannot lose any more symmetry. The groups $D_{1}^{2 j-1}$ and $D_{1}^{2 j}(j=1$ or 2$)$ represent bifurcation modes having one axis of line symmetry. Every

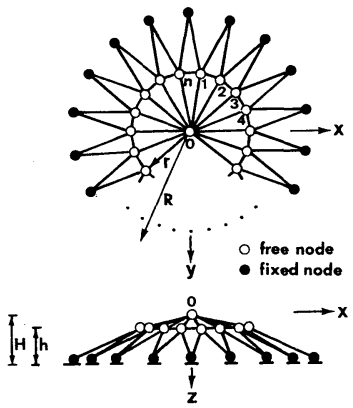

Fig. $1 \quad n$-Gonal Dome.

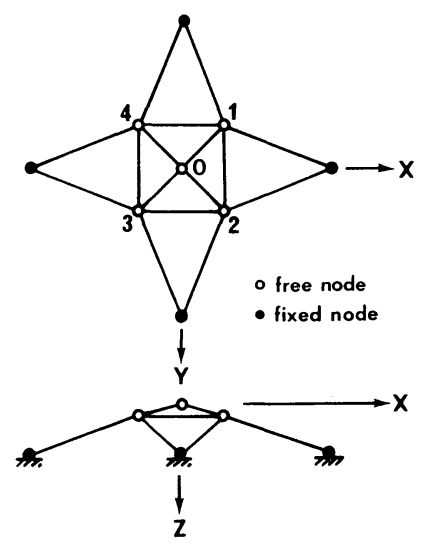

Fig. 2 4-Gonal Dome.

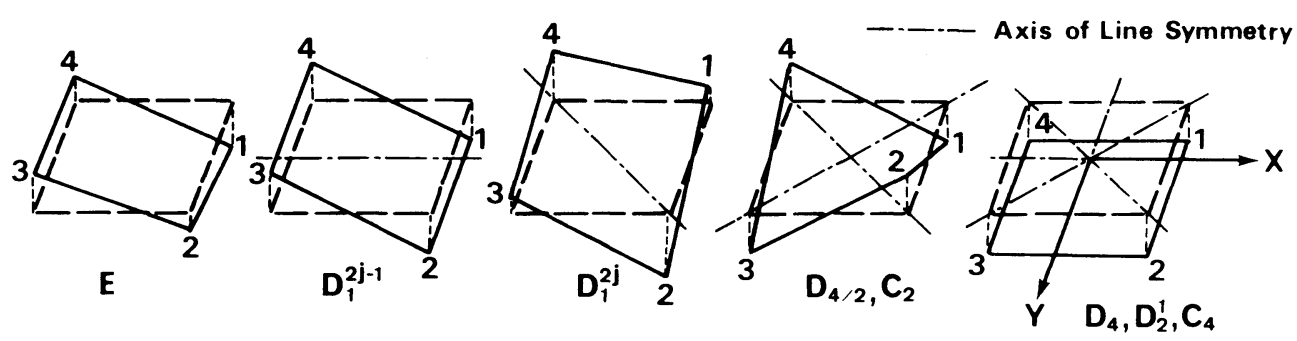

Fig. 3 Geometries of Bifurcation Modes of 4-Gonal Dome (Deformation of Nodes 1 through 4). 
other node displaces identically for the mode represented by the group $D_{4 / 2}$. The group $D_{4}$ expresses a completely symmetric deformation mode, which is not associated with a bifurcation path but with a fundamental path.

Next, potential bifurcation modes of the polygonal domes with degrees of three through 16 were obtained in this fashion. Table 1 shows the existence of these modes, where the symbol $(\bigcirc)$ expresses the subgroups which have relevant bifurcation modes and $(X)$ denotes those not having relevant modes due to mode degenerations.

The subgroups of the group $D_{n}$ used to represent these modes can be classified into the seven general types $E, C_{i}, D_{i}^{j}$, $D_{i}^{2 j-1}, D_{i}^{2 j}, D_{n / 2}$ and $D_{n}$, which are defined as follows:

$$
\begin{aligned}
& E=\left\langle\sigma_{1}\right\rangle \\
& C_{i}=\left\langle\sigma_{1}, \sigma_{1+n / i}, \cdots, \sigma_{1+n(i-1) / i}\right\rangle \\
& D_{i}^{j}=\left\langle\sigma_{1}, \sigma_{1+n / i}, \cdots \sigma_{1+n(i-1) / i}, \quad \tau \sigma_{j}, \quad \tau \sigma_{j+n / i}, \cdots \tau \sigma_{j+n(i-1) / i}\right\rangle \\
& D_{i}^{2 j-1}=\left\langle\sigma_{1}, \sigma_{1+n / i}, \cdots \sigma_{1+n(i-1) / i}, \tau \sigma_{2 j-1}, \tau \sigma_{2 j-1+n / i}, \cdots \tau \sigma_{2 j-1+n(i-1) / i}\right\rangle \\
& D_{i}^{2 j}=\left\langle\sigma_{1}, \sigma_{1+n / i}, \cdots \sigma_{1+n(i-1) / i}, \quad \tau \sigma_{2 j}, \quad \tau \sigma_{2 j+n / i}, \cdots \tau \sigma_{2 j+n(i-1) / i}\right\rangle \\
& D_{n / 2}=\left\langle\sigma_{1}, \sigma_{3}, \cdots \sigma_{n-1}, \tau \sigma_{2}, \tau \sigma_{4}, \cdots \tau \sigma_{n}\right\rangle \\
& D_{n}=\left\langle\sigma_{1}, \sigma_{2}, \cdots \sigma_{n}, \tau \sigma_{1}, \tau \sigma_{2}, \cdots \tau \sigma_{n}\right\rangle
\end{aligned}
$$

\begin{tabular}{|c|c|c|c|c|c|c|c|c|c|c|c|c|c|c|}
\hline \multirow{2}{*}{ Modes } & \multicolumn{14}{|c|}{ Degrees } \\
\hline & 3 & 4 & 5 & 6 & 7 & 8 & 9 & 10 & 11 & 12 & 13 & 14 & 15 & 16 \\
\hline E & $\mathrm{O}$ & 0 & 0 & $\mathrm{O}$ & $\mathrm{O}$ & 0 & $\mathrm{O}$ & 0 & 0 & $\mathrm{O}$ & $\mathrm{O}$ & $\mathrm{O}$ & $\mathrm{O}$ & 0 \\
\hline $\begin{array}{l}C_{2} \\
C_{3} \\
C_{4} \\
C_{5}\end{array}$ & & $x$ & $x$ & $\begin{array}{l}0 \\
x\end{array}$ & & $\begin{array}{l}0 \\
\times\end{array}$ & $\mathrm{O}$ & $\begin{array}{l}0 \\
\times\end{array}$ & & $\begin{array}{l}0 \\
0 \\
0\end{array}$ & & $\mathrm{O}$ & $\begin{array}{l}0 \\
0\end{array}$ & $\begin{array}{l}0 \\
0\end{array}$ \\
\hline $\begin{array}{l}D \\
D \\
D \\
D, \\
D \\
D \\
D \\
D\end{array}$ & 0 & & 0 & 0 & 0 & & $\begin{array}{l}0 \\
0\end{array}$ & O & O & 0 & 0 & 0 & $\begin{array}{l}0 \\
0 \\
0\end{array}$ & \\
\hline $\begin{array}{l}D_{1\}-1}^{2 j-1} \\
D_{1}^{2 j}\end{array}$ & & $\begin{array}{l}\text { O } \\
\text { O }\end{array}$ & & $\begin{array}{l}\mathrm{O} \\
\mathrm{O}\end{array}$ & & $\begin{array}{l}\mathrm{O} \\
\mathrm{O}\end{array}$ & & $\begin{array}{l}\mathrm{O} \\
\mathrm{O}\end{array}$ & & $\begin{array}{l}0 \\
0\end{array}$ & & $\begin{array}{l}\mathrm{O} \\
\mathrm{O}\end{array}$ & & $\begin{array}{l}0 \\
0\end{array}$ \\
\hline $\begin{array}{l}D_{2}^{2 j-1} \\
D_{2}^{2 j}\end{array}$ & & $\begin{array}{l}x \\
x\end{array}$ & & & & $\begin{array}{l}0 \\
0\end{array}$ & & & & $\begin{array}{l}\mathrm{O} \\
\mathrm{O}\end{array}$ & & & & $\begin{array}{l}\mathrm{O} \\
\mathrm{O}\end{array}$ \\
\hline $\begin{array}{l}D_{3}^{2 f-1} \\
D_{3}^{2 f}\end{array}$ & & & & $\begin{array}{l}x \\
x\end{array}$ & & & & & & $\begin{array}{l}0 \\
0\end{array}$ & & & & \\
\hline $\begin{array}{l}D_{4}^{2 j-1} \\
D_{4}^{2 j}\end{array}$ & & & & & & $\begin{array}{l}x \\
x\end{array}$ & & & & & & & & $\begin{array}{l}\mathrm{O} \\
\mathrm{O}\end{array}$ \\
\hline $\begin{array}{l}D_{n / 2} \\
D_{n} \\
\end{array}$ & $\mathrm{O}$ & $\begin{array}{l}0 \\
0 \\
\end{array}$ & 0 & $\begin{array}{l}0 \\
0\end{array}$ & 0 & $\begin{array}{l}\text { O } \\
0 \\
\end{array}$ & $\mathrm{O}$ & $\begin{array}{l}0 \\
0\end{array}$ & 0 & $\begin{array}{l}\mathrm{O} \\
\mathrm{O} \\
\end{array}$ & $\mathrm{O}$ & $\begin{array}{l}0 \\
0 \\
\end{array}$ & $\mathrm{O}$ & $\begin{array}{l}0 \\
0 \\
\end{array}$ \\
\hline $\begin{array}{l}\mathrm{Bi} \\
\mathrm{Bif}\end{array}$ & & $\begin{array}{l}\text { on } \\
\text { on }\end{array}$ & & & & & & & & & & & & \\
\hline$\sigma_{j+n(i-1}$ & & & & & & & & $j=1$ & , & $\cdot, r$ & $/ i$ & & & \\
\hline$n / i, \cdot \cdot$ & $\tau \sigma_{2 j}$ & $-1+$ & $n i-$ & $1 / i\rangle$ & & & & $j=1$ & , 2 & $\cdot \cdot r$ & $12 i$ & & & \\
\hline$\tau \sigma_{2 j+r}$ & $-1) /$ & & & & & & & $j=1$ & , 2, & $\cdot \cdot n$ & $12 i$ & & & \\
\hline
\end{tabular}

Table 1 Potential Bifurcation Modes of Polygonal Domes.

Some of these modes may not exist in actual analytical behavior of certain domes; however, the modes should cover all the possible bifurcation modes without fail. The presence of element $\sigma_{1+n k / i}$ in a group denotes that the mode related to this group is symmetric about the origin $\mathrm{O}$ and can be preserved by a $360 \cdot k / i$ degree rotation about the origin ; the presence of $\tau \sigma_{1+n k / i}$ indicates that the mode is symmetric in the straight line which intersects with the $y$-axis at the origin at an angle of $-180 \cdot k / i$ degrees. Geometric configurations of some of these modes are schematically illustrated in Fig. 4, where the symbols $(\bigcirc),(\triangle)$... denote that the nodes having the same symbol displace identically.

The trivial group $E$ represents a completely asymmetric deformation mode, which exists for all kinds of degrees. The mode has the most unsymmetric configuration among the bifurcation modes since the group has the smallest order among the subgroups.

Bifurcation modes represented by the group $C_{i}(i=2,3, \cdots)$ exist for the domes with degrees which can be factorized by the number $i$, except for the case where a mode diminishes due to a mode degeneration (see Table 1). As can be seen from Fig. 4, these modes are symmetric about the origin since the group $C_{i}$ is made up of a series of rotations $\sigma_{j}$.

The modes represented by groups $D_{i}^{2 j-1}$ and $D_{i}^{2 j}$ exist for the domes whose degrees can be factorized by the number $2 i$. The modes related to the group $D_{i}^{j}$ exist for the domes whose degrees cannot be factorized by the number $2 i$ but factorized by the number $i$. As can be seen from Fig. 4, these bifurcation modes exhibit both point and line symmetries owing to the presence of the elements $\sigma_{j}$ and $\tau \sigma_{j}$ in the groups, except for the case of $i=1$ where the modes have only line-symmetric nature.

Bifurcation modes represented by the group $D_{n / 2}(n=4,6,8, \cdots)$ exist for the polygonal domes with 


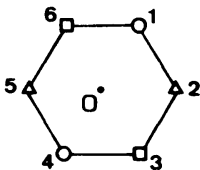

$C_{2}(n=6)$

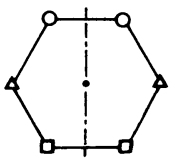

$D_{1}^{2 j-1}$

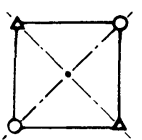

$D_{4 / 2}$

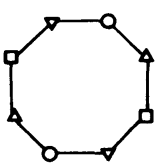

$C_{2}(n=8)$
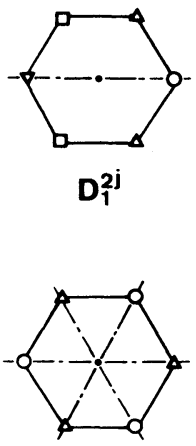

$D_{6 / 2}$

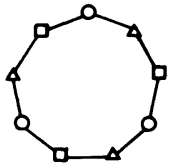

$C_{3}(n=9)$

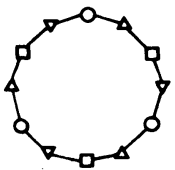

$C_{3}(n=12)$

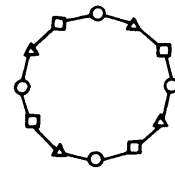

$C_{4}(n=12)$
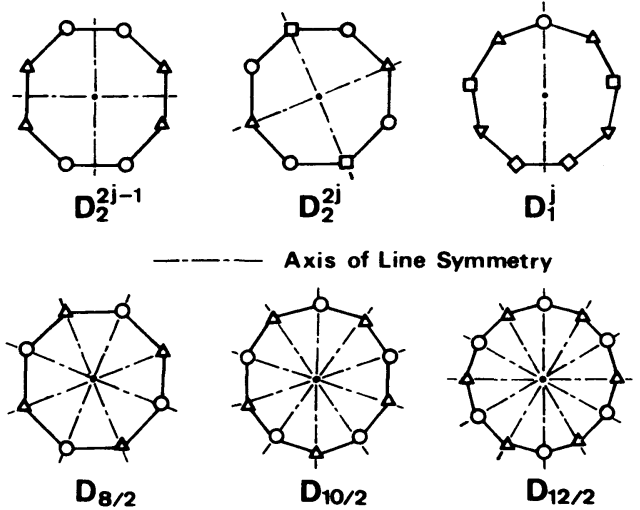

Fig. 4 Geometries of Typical Bifurcation Modes.

even-numbered degrees. These modes, which exhibit an identical displacement for every other node (see Fig. 4), have the highest-leveled point and line symmetries among the bifurcation modes because of their greatest order, $n$. Accordingly they should play an important role in determining load carrying capacities of dome structures.

The group $D_{n}$ represents a perfectly symmetric deformation mode, which exists for all degrees. This mode, related to the fundamental path, is not concerned with bifurcation behavior but with pre-bifurcation behavior.

As illustrated by these examples, the existence of bifurcation modes is highly periodic with respect to the degrees. For example, the mode represented by the group $D_{1}^{j}$ exists for odd degrees, whereas do those by $D_{1}^{2 j-1}$ and $D_{1}^{2 j}$ for even degrees. Such a feature can be well explained by Lagrange's theorem, which states that the order of a group is divided by the order of its subgroup ${ }^{12)}$. The order of the dihedral group $D_{n}$, equal to $2 n$, has the degree $n$ as a factor so that the bifurcation behavior covariant with this group should be greatly influenced by the way how the degree is factorized. For example, in the case where the degree is a prime number, the order $2 n$ has only three factors, 1,2 , and $n$. Otherwise, the order has more than three factors in association with the factors of the degree $n$.

As can be expected from these discussions, the bifurcation behavior of a polygonal dome is greatly influenced by the way how the degree is resolved into factors. Such an expectation can be insured from the hierarchal structures of bifurcation modes shown in Table 2 (The symbol ' $S \rightarrow T \rightarrow U$ ' used in this table indicates that the group $T$ is a subgroup of the group $S$ and $U$ is of $T$; moreover, one can bypass the group $T$ and interpret that $U$ is a subgroup of $S$ ). As can be seen, the degrees having the same factorization exhibited the same hierarchy. Based on this feature, the degrees three through 16 can be divided into the following eight general categories : $p, 2^{2}, p^{2}, 2 \cdot p, p \cdot q, 2^{3}, 2^{2} \cdot p$, and $2^{4}$, where the variables $p$ and $q$ denote the prime numbers other than ' 2 '. During the course of this categorization, the factor ' 2 ' played different roles than other factors did.

A strong correlationship existed between the complexity of hierarchal structures and the categories. Polygonal domes with a prime degree $p(3,5,7,11$ and 13), which have only three types of bifurcation modes, exhibited a very simple bifurcation mode hierarchy. By contrast, those with non-prime degrees 
Table 2 Hierarchal Frameworks of Bifurcation Modes.

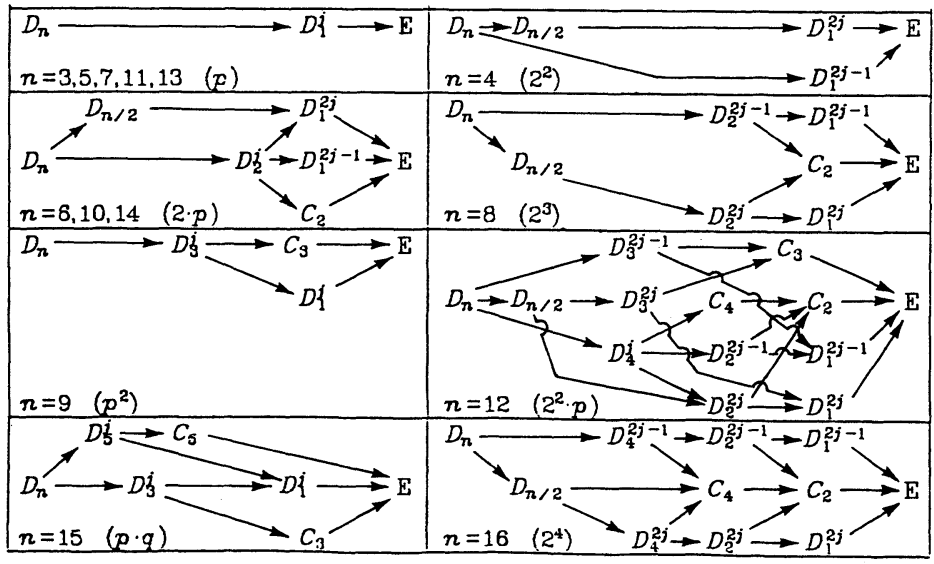

had much more complex hierarchies.

As the factorization of the degrees tended to be more complex from $2^{2}$ through $2^{3}$ to $2^{4}$, the correspondent hierarchies became increasingly complex in a systematic manner. The growth of the hierarchal framework was so rapid that the dome with a degree $2^{m}$ ( $m$ is a large integer) should exhibit an extremely huge framework. However, the recurrent properties of the frameworks (see Fig. 5) can be of great assistance in studying the behavior. For example, the hierarchy for the degree $2^{m}$ can be

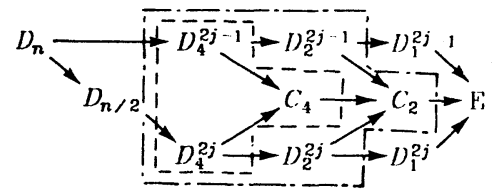

- Removed for $n=8$

- - Removed for $n=4$

Fig. 5 Recurrent Properties of Hierarchies $\left(n=2^{2}, 2^{3}\right.$, and $\left.2^{4}\right)$. determined by referring to the hierarchies for small degrees (e. g. , $2^{2}$ and $2^{3}$ ) and constructing recurrence formulae.

The hierarchy also became complex for the case where a degree was composed of multiple prime numbers. For example, the domes with degrees 6,10 , and 14 , which belong to the category $2 \cdot p$, had greater number of bifurcation mode types and a more complex hierarchy than the dome belonging to the category $2^{2}$ did. Since the level of symmetry of polygons is directly proportional to the complexity of the factorization, the complexity of bifurcation mode hierarchy of polygons is enhanced with increasing symmetry. The concept of symmetry should play an important role in describing bifurcation buckling behavior, to be interpreted as a process of losing symmetry ${ }^{10)} \cdot 11$.

These studies regarding bifurcation modes have demonstrated that the method for determining bifurcation modes proposed can advance potential bifurcation modes and their hierarchal frameworks in a methodical manner. The studies dealt with the bifurcation modes of polygonal-shaped domes with specific degrees in a heuristic manner so that it will be a natural course of future studies to make the conclusions drawn herein to be applicable to more general cases.

\section{ANALYTICAL BIFURCATION BEHAVIOR OF POLYGONAL DOMES}

In order to demonstrate the usefulness of the categories of degrees introduced in the previous section, bifurcation path tracing analyses were conducted on some of the polygonal domes. The finite displacement analysis technique and computer program for truss structures developed by Nishino et al. ${ }^{6}$ ) were employed for these analyses. The loading pattern listed in Table 3 was utilized. The parameters for defining the domes' geometries were specified as follows : $R=50, r=25, H=8.216$ and $h=6.216 \mathrm{~cm}$. Figure 6 compares external force versus crown displacement relationships (equilibrium paths) obtained for five-gonal and seven-gonal domes, whose degrees are both characterized by the category $p$. During the course of these analyses, deformation modes of these domes were investigated. This investigation has 
revealed that each bifurcation path can be characterized by one of the seven types of subgroups introduced in Eq. 3. In this figure, the bold-solid lines express the paths represented by the group $D_{n}$; the solid lines denote those by the group $D_{1}^{j}$ and so on. The symbol (O) expresses the bifurcation point with a single root, and $(\bigcirc)$ does that with a double root. As can be seen, both of these domes exhibited the same hierarchal structure made up of three types of bifurcation paths. Such a feature can be confirmed from Fig. 7, which shows equilibrium paths for the six-gonal and ten-gonal domes, whose degrees belong to the category $p \cdot q$. These domes also exhibited the same hierarchy with the same types of paths.

As can be seen, bifurcation processes among these sets of equilibrium paths follow perfectly the inter-group relationships listed in Table 2. For example, the paths $\mathrm{O}$ ), A), B) and C) follow the relationship $D_{n} \rightarrow D_{2}^{j} \rightarrow D_{1}^{2 j-1} \rightarrow E$. Of course, there are cases where certain subgroups in the relationships are bypassed. For example, the paths D), E) and F), represented by the group $D_{\mathrm{i}}^{2 j-1}$, branch directly from the fundamental paths represented by the group $D_{n}$ at the bifurcation points $\mathbf{a}, \mathbf{b}$, and $c$. In these cases, the group $D_{2}^{j}$ in that relationship is bypassed.

As we have seen, the categories introduced herein characterized the bifurcation properties. These categories would greatly contribute to the study of bifurcation modes. For example, a study of bifurcation modes of the dome with a small degree (e. g. , 2·3) should serve as a good reference in studying those for a large degree belonging to the same category (e.g., $2 \cdot 11$ and $2 \cdot 23$ ).

\section{TRACING BIFURCATION BEHAVIOR BY INITIAL IMPERFECTION TECHNIQUE}

Typical bifurcation modes of a series of simple truss domes were advanced in the previous section. In this section, these bifurcation modes are used to determine the initial imperfection modes which can initiate bifurcation buckling phenomena.

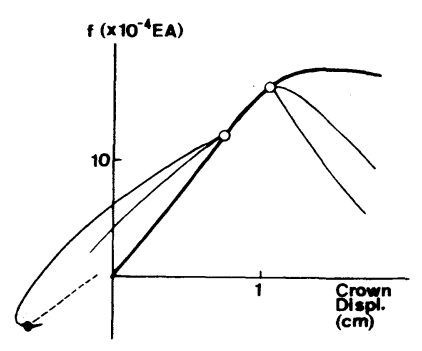

(a) 5-Gonal Dome

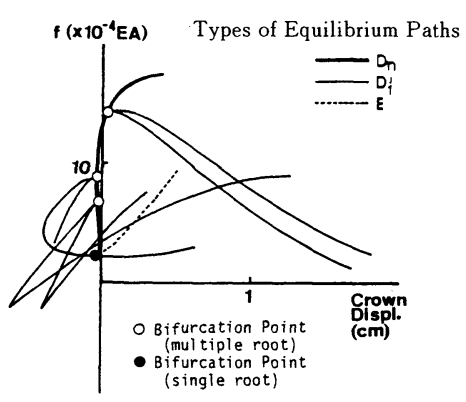

(b) 7-Gonal Dome

Fig. 6 Equilibrium Paths of 5-Gonal and 7-Gonal Domes (Category $p$ ).

Table 3 Loading Pattern (Vertically Applied at Nodes 0 to $n$ )

\begin{tabular}{|c|c|}
\hline $\begin{array}{c}\text { Node } \\
\text { Number }\end{array}$ & $\begin{array}{c}\text { Loading } \\
\text { Paltern }\end{array}$ \\
\hline 0 & 0.5 \\
1 & 1.0 \\
2 & 1.0 \\
3 & 1.0 \\
. &. \\
. &. \\
. &. \\
$\mathrm{n}$ & 1.0 \\
\hline
\end{tabular}

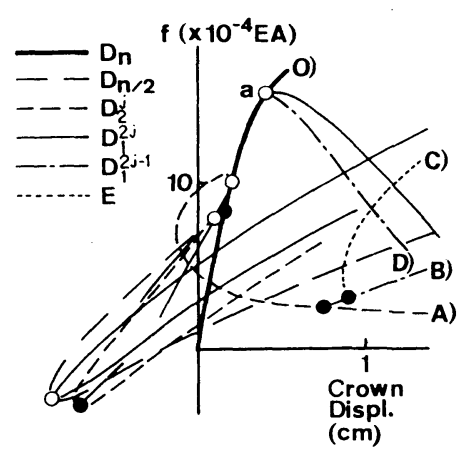

(a) 6-Gonal Dome

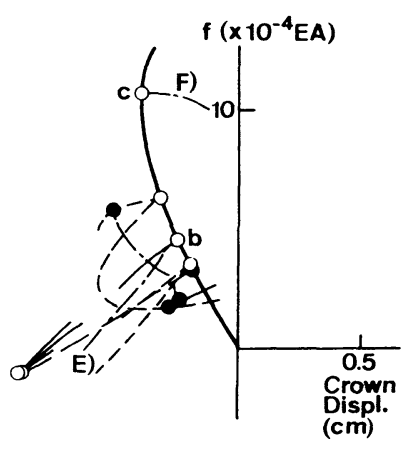

(b) 10-Gonal Dome

Fig. 7 Equilibrium Paths of 6-Gonal and 10-Gonal Domes (Category $2 \cdot p$ ). 
An elastic, eight-gonal, truss dome (see Fig. 8) subjected to the symmetric loading pattern listed in Table 3 is used here as a numerical example. The bifurcation behavior of this dome is covariant with the group $D_{8}$.

As we have seen in Table 1, this dome has the following seven types of bifurcation modes, represented by the subgroups $E, C_{2}, D_{1}^{2 j-1}, D_{1}^{2 j}, D_{2}^{2 j-1}, D_{2}^{2 j}$, and $D_{8 / 2}$. Figure 9 schematically illustrates typical geometries of these bifurcation modes. In addition to this qualitative information regarding bifurcation modes, the following theoretical finding ${ }^{5}$ ) was used to determine the initial imperfections : the external load vector $f$ is orthogonal to the critical bifurcation mode vector $d x$ at a bifurcation point, that is,

$d x \cdot f=0$

where the symbol $(\cdot)$ denotes the inner product.

On the basis of these features, normalized initial imperfection modes were selected as listed in Table 4. The imperfection modes for the groups $D_{8 / 2}$ and $D_{2}^{2 j-1}$ were uniquely determined from the features ; however, such was not the case for the modes for the other groups owing to redundant number of degree of freedoms involved. These modes were specified in such a way that their components have the simplest integer ratios as possible.

The initial imperfection modes were scaled by a constant value $0.01 \mathrm{~cm}$ and the $z$-direction coordinates of the nodes 1 through 8 of the dome were altered according to these scaled modes. Figure 10 compares the equilibrium paths of the dome obtained by using these initial imperfections and those obtained for the perfect configuration of the dome. As can be seen from this figure, the initial imperfections evaluated in this manner offered us excellent approximations of bifurcation buckling capacities of the dome.

The imperfection modes and the actual bifurcation modes are compared in Table 4. These two types of modes perfectly matched

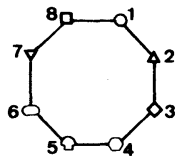

$E$

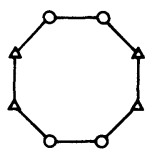

$D_{2}^{2 j-1}$

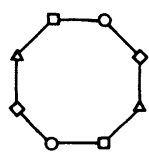

$\mathrm{C}_{2}$

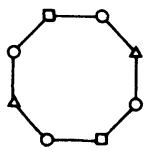

$\mathrm{D}_{2}^{2 \mathrm{j}}$
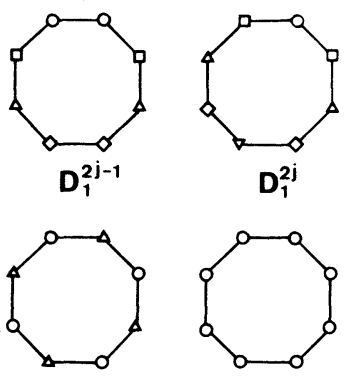

$D_{8 / 2}$

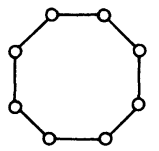

$D_{8}$
Fig. 9 Geometries of Bifurcation Modes of 8 -Gonal Dome.

Table 4 Comparison of Initial Imperfection and Bifurcation Modes.

\begin{tabular}{|c|c|c|c|c|c|c|c|c|c|c|c|c|c|c|c|c|}
\hline \multirow{3}{*}{$\begin{array}{l}\text { Mode } \\
\text { Type }\end{array}$} & \multicolumn{8}{|c|}{ Initial Imperfection Modes Used } & \multicolumn{8}{|c|}{ Bifurcation Modes Obtained } \\
\hline & \multicolumn{16}{|c|}{ Node Number } \\
\hline & 1 & 2 & 3 & 4 & 5 & 6 & 7 & 8 & 1 & 2 & 3 & 4 & 5 & 6 & 7 & 8 \\
\hline$D_{n / 2}$ & 1 & -1 & 1 & -1 & 1 & -1 & 1 & -1 & 1 & -1 & 1 & -1 & 1 & -1 & 1 & -1 \\
\hline$D_{2}^{2 j-1}$ & 0 & 1 & 1 & 0 & 0 & -1 & -1 & 0 & 0 & 1 & 1 & 0 & 0 & -1 & -1 & 0 \\
\hline$D_{2}^{2 j}$ & 0 & 1 & 0 & -1 & 0 & 1 & 0 & -1 & 0 & 1 & 0 & -1 & 0 & 1 & 0 & -1 \\
\hline$D_{1}^{2 j-1}$ & 2 & 1 & -1 & -2 & -2 & -1 & 1 & 2 & 2 & -5 & 5 & -2 & -2 & 5 & -5 & 2 \\
\hline$D_{1}^{2 j}$ & 0 & 1 & 2 & 1 & 0 & -1 & -2 & -1 & 0 & -3 & 5 & -3 & 0 & 3 & -5 & 3 \\
\hline
\end{tabular}

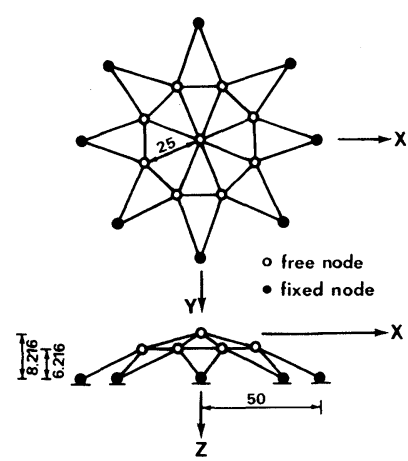

Fig. 8 -Gonal Dome (unit in $\mathrm{cm}$ ).

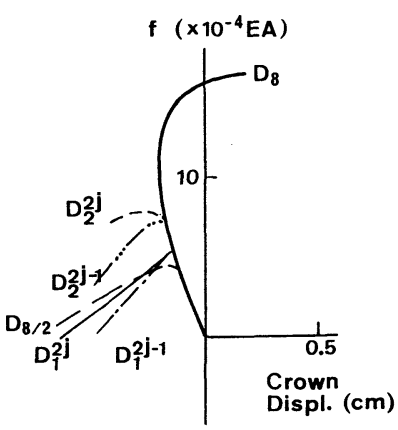

(a) Initial Imperfection

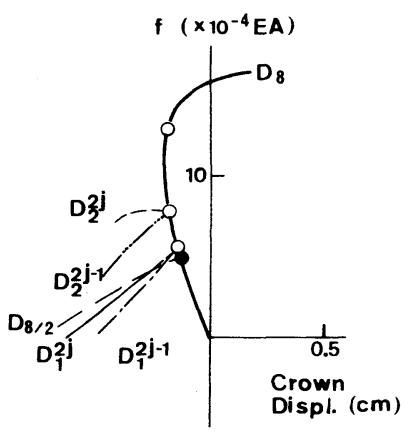

(b) Perfect Geometry

Fig. 10 Tracing of Bifurcation Behavior Using Initial Imperfections. 
for the groups $D_{8 / 2}, D_{2}^{2 j-1}$ and $D_{2}^{2 j}$. The two modes were not in good accordance for the groups $D_{1}^{2 j-1}$ and $D_{1}^{2 j}$, which had rather more complex bifurcation modes. However, as we have seen in Fig. 10, such a drawback did not limit the usefulness of the imperfection modes in estimating the bifurcation buckling capacities.

With the aid of the information regarding bifurcation modes, the initial imperfection technique will enable one to efficiently obtain bifurcation buckling capacities of domes with much more confidence since the number of imperfection modes to be attempted is greatly reduced from the information. Such information would be of great practical assistance in determining bifurcation buckling capacities of dome structures.

\section{SUMMARY AND CONCLUSIONS}

This paper has advanced a study of potential bifurcation modes of truss dome structures. Dihedral (symmetry) groups, extensively used to describe the symmetry of regular polygons in mathematics ${ }^{10)} \cdot 11$, were suggested to be used as a mathematical tool for investigating the bifurcation modes. Fujii's findings with regard to the system covariant with a symmetry group were introduced and explained in detail. This explanation clarified the interrelationships between the bifurcation behavior and symmetry groups.

Potential bifurcation modes of a series of polygonal-shaped truss domes were determined from the investigation of the subgroups of dihedral groups, which are symmetry groups of regular polygons. The bifurcation modes were classified into seven general types and the existence of the modes was found to be highly periodic with regard to the degree of polygons.

The degrees three through 16 were divided into eight general categories through the investigation of hierarchal structures of bifurcation modes. The domes with degrees belonging to the same category had the same types of bifurcation modes and hierarchy. The domes with prime degrees, belonging to the category $p$, had only three bifurcation modes, thereby holding very simple bifurcation mode structures. By contrast, the domes with degrees belonging to the other categories had significantly increased number of bifurcation modes, especially for the case where the degrees had complex factorizations. As the factorization of degrees became complex, the hierarchy grew huge and complex. The concept of categories, which can characterize the bifurcation phenomena, would greatly contribute to the investigation of bifurcation modes. A study of the modes of the dome with a small degree could serve as a good reference in studying those for a large degree belonging to the same category. Such an expectation was verified through an investigation of analytical bifurcation behavior of polygonal domes.

The level of symmetry of polygons had a strong correlation with the complexity of the factorization of degrees. The complexity of bifurcation mode hierarchy of polygons was enhanced with increasing symmetry associated with an increase in the number of bifurcation modes.

The information regarding bifurcation modes advanced in this manner was employed to determine initial imperfection modes. A series of analyses were performed on an eight-gonal truss dome with the use of the imperfection modes determined on the basis of the information. The analytical results obtained were capable of accurately approximating bifurcation buckling capacities of the dome in an efficient and methodical manner owing to a great reduction of the number of bifurcation modes to be attempted. This procedure for determining initial imperfections will be valuable in evaluating load carrying capacities of dome type structures undergoing bifurcation buckling phenomena.

Most of these conclusions drawn herein are based on an investigation of bifurcation modes of polygonal domes with specific degrees performed in a heuristic fashion so that verification of the conclusions for more general cases remains to be settled in the future.

\section{ACKNOWLEDGEMENT}

The authors are grateful to Professor Fumio Nishino for his contributions to this research paper. 


\section{REFERENCES}

1) Yamada, M. and Yamada, S. : Experimental and theoretical studies of the non-linear deflection behavior of clamped shallow spherical shells under concentrated loads, Transactions of the Architectural Institute of Japan, AIJ, No. 321, pp.73-81, November 1982 (in Japanese).

2) Yoshida, Y., Masuda, N. and Matsuda, T. : A discrete element approach to elasto-plastic large displacement analysis of thin shell structures, Proceedings of Japan Society of Civil Engineers, No.288, pp. 41-55, August 1979 (in Japanese).

3) Fukumoto, Y. and Mizuno, E. : Static and quasi-static stability and imperfection sensitivity of reticulated shell structures, Proceedings of Japan Society of Civil Engineers, No. 288, pp. 29-40, August 1979 (in Japanese).

4) Hosono, T. : Analysis of elastic buckling problem by arc length method, Part 1, The nature of incremental solution at the buckling point, Transactions of the Architectural Institute of Japan, AIJ, No.242, pp.41-48, April 1976 (in Japanese).

5) Hosono, T. : Analysis of elastic buckling problem by arc length method, Part 2, The arc length method for actual numerical analysis, Transactions of the Architectural Institute of Japan, AIJ, No. 243, pp. 21-30, May 1976 (in Japanese).

6) Nishino, F., Ikeda, K., Sakurai, T. and Hasegawa, A. : A total lagrangian nonlinear analysis of elastic trusses, Proceedings of Japan Society of Civil Engineers, Structural Eng./Earthquake Eng. No.344, I-1, pp. 39-53, April 1984.

7) Endou, A., Hangai, Y. and Kawamata, S. : Post-buckling analysis of elastic shells of revolution, Report of Inst. Industrial Science, 26, The University of Tokyo, 1976.

8) Ikeda, K. : Proc. of the 40-th Annual Meeting of Japan Society of Civil Engineers, pp. 83-84, September 1985 (in Japanese).

9) Ikeda, K., Matushita, S. and Torii, K. : Symmetry breaking bifurcation behavior of dome structures and group theory, Proceedings of Japan Society of Civil Engineers, Structural Eng. /Earthquake Eng., Vol. 3, No. 1, pp. 277-286, April 1986.

10) Fujii, H. : Striped patterns in the nature, Nonlinear phenamena and analyses, Introduction to modern mathematics (1), A Special Issue of Mathematics Seminar, October 1979 (in Japanese).

11) Fujii, H. : Tracing global bifurcation phenomena, Numerical analyses and nonlinear phenomena, Introduction to modern mathematics (2), A Special Issue of Mathematics Seminar, November 1981 (in Japanese).

12) Baumslag, B. and Chandler, B. : Theory and problems of group theory, Outline Series in Mathematics, McGrow-Hill Book Company, June 1968.

(Received November 5 1985) 